\title{
Thorium-Reactor Physics (Session 8)
}

\author{
Robert Cywinski
}

The deployment of thorium as a nuclear fuel has had a long and largely successful history, stretching back to the 1960 s when the thorium-fueled Peach Bottom nuclear plant pioneered the commercial exploitation of high-temperature gas reactor technology. However, since the closure of the thorium-fueled 300 MW HTGR in Germany in the late 1980s, thorium reactor physics has been largely neglected, except perhaps in India. We now appear to be on the brink of a considerable resurgence of interest in thorium as a potential nuclear fuel and, coupled with the development of new reactor designs and modes of operation, this has presented new opportunities, but also many new challenges. Session 8 of IThEC focused upon both the opportunities and challenges.

Firstly, Srinavasan Ganesan highlighted the considerable breadth of activities of the Nuclear Data Physics Centre of India with a clear emphasis on the need for and progress in the measurement and curation of neutron and charged particle induced cross sections associated with the Th- $U$ fuel cycle and other processes, particularly with a view to improving the reliability of Monte Carlo codes for reactor design and simulation.

This theme was continued by Frank Gunsing (CEA) who provided a comprehensive overview of the exploitation of phases I and II n_TOF (neutron time of flight) facility at CERN between 2001 and 2012 for the measurement of capture and fission cross sections relevant to both the thorium and uranium fuel cycles and the transmutation of nuclear waste.

Konstantin Mikityuk (PSI) followed with a presentation on the history and technology of fast reactors whilst exploring factors such as sustainability, safety, proliferation resistance, and radiotoxicity of the waste associated with the thorium fuel cycle. Although calculations show that in principle an equilibrium closed cycle fast reactor fueled with thorium is possible, the thorium cycle has both advantages and disadvantages. Particular advantages are the very low quantity of minor actinides in the EQL-Th fuel cycle; the fact that the Doppler effect is stronger and void effect are weaker in EQL-Th fuel compared with EQL-U; and the radiotoxicity and decay heat of EQL-Th fuel are lower during the first 10,000 years of cooling compared with EQL-U fuel. However, the disadvantages include the fact that U-232, the precursor of hard gamma emitters, is produced in the Th-U cycle with the $(n, 2 n)$ reaction being higher in a fast spectrum; $k_{\infty}$ of equilibrium fuel is lower for the Th-U cycle compared with $\mathrm{U}-\mathrm{Pu}$, so to reach iso-breeding, blankets of fertile material are required; and the fissile precursor of U-233 (Pa-233) has a longer half-life compared with $\mathrm{Np}-239$, so the potential for separation and decay to pure U-233 is higher. Moreover, it was emphasized that our collective experience with thorium fuels and thorium reactor technology is significantly less than that with U-Pu fuel cycles and deployment.

The final presentation of the session was made by Elsa Merle-Lucotte (IN2P3, CNRS), who gave an introduction to the physics of molten salt fast reactors (MSFRs). Not only were the advantages of a liquid fuel considered, but the particular advantages of the Th- ${ }^{233} \mathrm{U}$ cycle over the U-P cycle (for which the neutronic properties of $\mathrm{F}$ in the LiF salt are not favorable) were also demonstrated. There then followed a detailed evaluation of the design characteristics, neutronics, fueling, and safety of a realistic MSFR, carried out with support from the Euratom-funded EVOL program.

In the ensuing discussion it was widely accepted that to move the thorium agenda forward there is a need for a continuing international effort to measure, refine, and curate nuclear data related to the thorium cycle. Additionally, deep modeling of whole thorium-fueled reactor systems using that data must be carried out, and this in turn necessitates a willingness to accept or reject appropriate or inappropriate thorium deployment scenarios based upon evidence provided by the data and the modeling.

R. Cywinski $(\square)$

University of Huddersfield, West Yorkshire, HD1 3DH, UK 